\title{
The analgesic effect of rolipram is associated with the inhibition of the activation of the spinal astrocytic JNK/CCL2 pathway in bone cancer pain
}

\author{
CHI-HUA GUO $^{1 *}$, LU BAI $^{2 *}$, HUANG-HUI WU ${ }^{3}$, JING YANG $^{4}$, \\ GUO-HONG CAI ${ }^{5}$, XIN WANG ${ }^{5}$, SHENG-XI WU $^{5}$ and WEI MA ${ }^{1}$
}

\begin{abstract}
Departments of ${ }^{1}$ Orthopedics, and ${ }^{2}$ Medical Imaging, The First Affiliated Hospital of Xi'an Jiaotong University, Xi'an, Shaanxi 710061; ${ }^{3}$ Department of Anesthesiology, Fuzhou General Hospital of Nanjing Military Region, Fuzhou, Fujian 350025; ${ }^{4}$ Institute of Neuroscience, School of Medicine, Zhejiang University, Hangzhou, Zhejiang 310027;

${ }^{5}$ Department of Neurobiology and Collaborative Innovation Center for Brain Science, The Fourth Military Medical University, Xi'an, Shaanxi 710032, P.R. China
\end{abstract}

Received June 4, 2016; Accepted September 19, 2016

DOI: $10.3892 /$ ijmm.2016.2763

\begin{abstract}
Bone cancer pain (BCP) is one of the most difficult and intractable tasks for pain management, which is associated with spinal 'neuron-astrocytic' activation. The activation of the c-Jun N-terminal kinase (JNK)/chemokine (C-C motif) ligand (CCL2) signaling pathway has been reported to be critical for neuropathic pain. Rolipram (ROL), a selective phosphodiesterase 4 inhibitor, possesses potent anti-inflammatory and anti-nociceptive activities. The present study aimed to investigate whether the intrathecal administration of ROL has an analgesic effect on BCP in rats, and to assess whether the inhibition of spinal JNK/CCL2 pathway and astrocytic activation are involved in the analgesic effects of ROL. The analgesic effects of ROL were evaluated using the Von Frey and Hargreaves tests. Immunofluorescence staining was used to determine the number of c-Fos immunoreactive neurons, and the expression of spinal astrocytes and microglial activation on day 14 after tumor cell inoculation. Enzyme-linked immunosorbent assay (ELISA) was
\end{abstract}

Correspondence to: Professor Wei Ma, Department of Orthopedics The First Affiliated Hospital of Xi'an Jiaotong University, 277 West Yanta Road, Xi'an, Shaanxi 710061, P.R. China

E-mail: moon007qq@163.com

Professor Sheng-Xi Wu, Department of Neurobiology and Collaborative Innovation Center for Brain Science, The Fourth Military Medical University, 169 Chang Le Xi Road, Xi'an, Shaanxi 710032, P.R. China E-mail: 821496575@qq.com

*Contributed equally

Abbreviations: $\mathrm{BCP}$, bone cancer pain; ROL, rolipram; SDH, spinal dorsal horn; GFAP, glial fibrillary acidic protein; JNK/CCL2 pathway, c-Jun N-terminal kinase/chemokine (C-C motif) ligand 2 pathway; IBA-1, ionized calcium binding adapter molecule-1

Key words: rolipram, analgesic, neuroglia, bone cancer pain, c-Jun $\mathrm{N}$-terminal kinase/chemokine (C-C motif) ligand 2 pathway pathway used to detect the expression of pro-inflammatory cytokines [interleukin (IL)-1 $\beta$, IL-6 and tumor necrosis factor (TNF)- $\alpha$ ] and chemokines (CCL2), and western blot analysis was then used to examine the spinal phosphodiesterase 4 (PDE4), ionized calcium binding adapter molecule-1 (IBA-1) and JNK levels on day 14 after tumor cell inoculation. The results revealed that ROL exerted a short-term analgesic effect in a dose-dependent manner, and consecutive daily injections of ROL exerted continuous analgesic effects. In addition, spinal 'neuron-astrocytic' activation was suppressed and was associated with the downregulation of spinal IL-1 $\beta$, IL- 6 and TNF- $\alpha$ expression, and the inhibition of PDE4B and JNK levels in the spine was also observed. In addition, the level of CCL2 was decreased in the rats with BCP. The JNK inhibitor, SP600125, decreased CCL2 expression and attenuated pain behavior. Following co-treatment with ROL and SP600125, no significant increases in thermal hyperalgesia and CCL2 expression were observed compared with the ROL group. Thus, our findings suggest that the analgesic effects of ROL in BCP are mainly mediated through the inhibition of 'neuron-astrocytic' activation, which occurs via the suppression of spinal JNK/CCL2 signaling.

\section{Introduction}

Many common types of cancer have a strong tendency to metastasize to the bones. Bone cancer pain (BCP) can be induced by tumor metastasis, and can occur at any time during the course of the disease. This severe and unmanageable pain severely affects the functional status and quality of life of patients $(1,2)$. Currently, the management of BCP is largely based on the classical 'analgesic ladder' that was promulgated by the World Health Organization in 1986. However, this traditional analgesic strategy is not satisfactory, and is also associated with significant side-effects (3). Hence, in BCP research, a major goal is to identify novel analgesic targets and therapies.

Currently, the cellular and molecular mechanisms underlying chronic pain have progressed from the idea of the dominant 'neuronal-based' theory to the idea of neuron- 
astrocyte interaction (4). Accumulating evidence has indicated that neuroglial cells, particularly astrocytes and microglial cells, further contribute to neurological disorders, including chronic pain $(5,6)$. Certain studies have suggested that spinal astrocytic activation depends on afferent neuronal inputs from the injured side and the release of chemical mediators. Both of pro-inflammatory cytokines, such as interleukin (IL)-1 $\beta$, IL-6, IL- 8 and tumor necrosis factor- $\alpha(\mathrm{TNF}-\alpha)$ that are released from activated neuroglia may provoke the cascading activation of adjacent neurons $(7,8)$, and then facilitate pain transmission through its coupling to neuronal glutamate receptors (9). This bidirectional neuron-neuroglial signaling plays a key role in astrocytic activation, cytokine production and the initiation and maintenance of allodynia and hyperalgesia (10). Recently, it has been demonstrated that the differential activation of mitogenactivated protein kinase (MAPK) family members occurs in spinal neuroglial cells following nerve injury and inflammation, and contributes to the generation and maintenance of chronic pain (11). Particularly, the spinal activation of c-Jun $\mathrm{N}$-terminal kinase (JNK) in astrocytes produces the initial factor chemokine (C-C motif) ligand (CCL2), which is pivotal for the maintenance of pain behavior (12).

Phosphodiesterase (PDE), which degrades the cyclic AMP (cAMP) and cyclic GMP (cGMP) phosphodiester bond, has the ability to terminate their action. There are at least 11 PDE subtypes, PDEs 5, 6 and 9 only hydrolyze cGMP, and PDEs 4, 7 and 8 only function on cAMP, whereas PDEs 1, 2, 3,10 and 11 hydrolyze both cAMP and cGMP (13). It has been demonstrated that PDE4 is mainly predominantly expressed in nerve and immune cells, and the inhibition of PDE4 in the central nervous system (CNS) has been shown to exert anti-inflammatory and anti-nociceptive effects (14). Previous studies have found that rolipram (ROL), one of the classical PDE4 inhibitors, exerts analgesic effects in inflammatory pain and neuropathic pain models (15-17), However, the analgesic mechanisms of action of ROL have not yet been fully clarified. Hence, in this study, we aimed to test the hypothesis that ROL attenuates pain behavior through the inhibition of spinal astrocytic activation via the JNK/CCL2 pathway in rats with BCP.

\section{Materials and methods}

Animal preparation. A total of 208 adult female Sprague-Dawley (SD) rats (8 weeks old, weighing 180-220 g) and 16 young female SD rats (3 weeks old, weighing 80-100 g) provided by the Experimental Animal Center of Xi'an Jiaotong university (Xi'an, China) were utilized in all the experiments. The adult SD rats were used to establish bone cancer pain models, and young female SD rats were used to culture tumor cells in abdominal cavity. All rats were housed in standard transparent plastic cages with a 12/12 h light/dark cycle (light on at 08:00 a.m.) under a $22-25^{\circ} \mathrm{C}$ ambient temperature with food and water ad libitum. Prior to being used in the experiments, animals were allowed to acclimatize to the housing environment for at least 7 days.

All experimental procedures received prior approval from the Animal Use and Care Committee for Research and Education of the Xi'an Jiaotong University and the National Institute for Physiological Sciences Animal Care and Use Committee. All efforts were made to minimize animal suffering and to reduce the number of animals used.
Cell line, doses and timings for drug administration. The $16 \mathrm{SD}$ rats (weighing, 80-100 g) received an intraperitoneal (i.p.) inoculation of Walker 256 mammary gland carcinoma cells $\left(2 \times 10^{6}\right.$ cells $/ \mathrm{ml}, 1 \mathrm{ml}$; CCL-38; ATCC, Manassas, VA, USA). After 1 week, the tumor cells were extracted from the cancerous ascitic fluid of the rats, and resuspended in a concentration of $1 \times 10^{7}$ cells $/ \mathrm{ml}$ in phosphate-buffered saline (PBS) for inoculation.

ROL (Sigma-Aldrich, St. Louis, MO, USA) was diluted with $0.9 \%$ saline containing a small amount of dimethyl sulfoxide (DMSO) prior to administrtion, and was injected in a volume of $10 \mu \mathrm{l}$ at the doses of $12.5,25,50$ and $100 \mu \mathrm{g} / \mathrm{kg}$. Previous studies have demonstrated the anti-inflammatory and anti-nociceptive effects of intraperitoneally and orally administered ROL in the CNS and in rodent models (14-17). However, in this study, ROL was administered via the intrathecal (i.t.) route due to the following reasons: first, PDE4, the target of ROL, is widely expressed in the CNS, including spinal neurons and glia (14). One of the advantages is that by i.t. administration, ROL can directly come in contact with its spinal target, PDE4. Second, the systemic administration of ROL has some side-effects, such as nausea and emesis; however, the i.t. injection largely reduces the dose of ROL and decreases the drug-related side-effects (18). This direct interaction would minimize the possible influence induced by systemic administration and the potential analgesic effect mediated by super-spinal mechanisms (11). Finally, spinal neurotoxicity is the main concern of the i.t. administration of ROL. Thus, we designed the rotarod test to identify the possible influence on motor function, and the results revealed that all rats were free from motor disabilities with the i.t. administration of ROL at the dose of 12.5-100 $\mu \mathrm{g} / \mathrm{kg}$ (p>0.05; Fig. 1D).

Similarly, the specific JNK inhibitor, SP600125 (S5567; Sigma-Aldrich), was also diluted with $0.9 \%$ saline containing a small amount of DMSO before application, and injected with $5 \mu \mathrm{g}$ in a volume of $10 \mu \mathrm{l}(12,19,20)$. SP600125 was administered daily from days 14 to 20 according to the study by $\mathrm{Hu}$ et al (21). They found that the spinal expression of p-JNK was time-dependently upregulated after tumor cell inoculation, and the increased exoression of p-JNK was first evident at day 7. Subsequently, it reached the peak at day 14, and was maintained at a high phosphorylation level up to day 21 . In order to better illustrate the connection between the JNK/CCL2 pathway and ROL, the determination of the optimal injection time point of SP600125 was performed at day 14. The same volume of ROL and SP600125 was injected to rats in both the Sham and BCP groups.

Yang et al demonstrated that mechanical allodynia and thermal hyperalgesia produced by tumor cell inoculation would reach the minimum on day 14; thus, the time points examined were days 2, 4, 7, 10, 12 and 14 in their study (5). For investigating the short-term analgesic effects of ROL, we designed the time points according to the study of Wu et al, which demonstrated that an effective analgesic effect could be observed at the minimum $6 \mathrm{~h}$ after single bolus; thus, we designed once/hour for detecting mechanical allodynia and thermal hyperalgesia (22). The time points of once/day from days 7 to 14 after tumor cell inoculation were designed for the long-term observation with a consecutive administration protocol according to Liu et al (23).

BCP model surgery. The inoculation was performed as previously described (5). Briefly, the rats were anaesthetized with 
chloral hydrate $(300 \mathrm{mg} / \mathrm{kg}$, i.p.), the right rear hindlimb was shaved in order to expose the skin over the femoral-tibial joint. The intercondylar eminence of the right tibia was exposed after cleaning the skin 3 times with iodine tincture and $75 \%$ ethanol. A 22 gauge needle was drilled into the site of place described previously (5), and a 20- $\mu 1$ microinjection syringe (Hamilton, Reno, NV, USA) containing a $10 \mu \mathrm{l}$ suspension of tumor cells, was used to slowly inject the tumor cells into the tibial cavity. The drilled hole was sealed with bone wax (Johnson \& Johnson, Rochester, NY, USA) in order to prevent the tumor cells from leaking outside the bone. For the sham-operated group, $10 \mu \mathrm{l}$ PBS was used to replace the tumor cells injected into the tibia.

The rats were divided into the following groups with 4 rats per group: i) the sham-operated group administered the vehicle ( $0.9 \%$ saline) (sham + vehicle); ii) the sham-operated group administered ROL (sham + ROL); iii) the rats with BCP administered the vehicle (BCP + vehicle); and iv) the rats with $\mathrm{BCP}$ administered ROL (BCP + ROL). For the initital determination of the optimal dose of ROL, and for the behavioral tests, we used 8 rats per group.

Intrathecal administration. Intrathecal administration was performed under chloral hydrate $(300 \mathrm{mg} / \mathrm{kg}$, i.p.) anesthesia. Briefly, a midline incision $(3 \mathrm{~cm})$ was cut on the backs of the rats at the level of the thoracic vertebrae. A pre-measured length of PE-10 tubing (ID $0.28 \mathrm{~mm}$ and OD $0.61 \mathrm{~mm}$ ) was passed caudally from the T8 to the L3 level of the spinal cord, fixed at the back of the rats' ears through subcutaneous tunnel, and $2 \mathrm{~cm}$ of the free end were exposed in the upper thoracic region. The rats were allowed to recover for a period of 3-5 days before being used in further experiments. Only animals that were judged to have no neurological deficits and that presented with complete paralysis of the tail and bilateral hind legs after the administration of $2 \%$ lidocaine $(10 \mu \mathrm{l})$ through the intrathecal catheter were used for the following experiments. The administration of ROL and/or SP600125 was administered intrathecally.

Behavioral test. The Von Frey test was applied to evaluate mechanical allodynia. Briefly, the rats were placed on an elevated wire-mesh floor in plexiglas chambers and the middle of the hindlimb paw was completely exposed. A series of Von Frey filaments were used to prod the plantar surface of the hindlimb paw with spaced increments ranging from 1 to $26 \mathrm{~g}$ (1, 2, 4, 6, 8, 10, 15 and 26 g; Stoelting Co., Wood Dale, IL, USA). The whole process of data recorded was determined according to the method and formula provided in the study by Dixon (24).

The Hargreaves test was applied to evaluate thermal hyperalgesia. In brief, the rats were placed individually in plexiglas chambers on an elevated glass floor. The plantar surface of the hindlimb paw was thermally stimulated by the radiant heat source. The withdrawal latencies were recorded according to previous studies (25). The threshold value of basal paw withdrawal latency (PWL) was approximately $16 \mathrm{sec}$. To prevent tissue thermal burn, the cut-off value was set at $20 \mathrm{sec}$. Finally, the blinding method was implemented strictly for the whole behavioral test (25).

Motor coordination was determined using a standard rat rotarod test (Shanghai Mobiledatum Information Technology Co., Ltd., Shanghai, China), as previously described (26). Daily training for each rat was performed at
30 min following the i.p. administration of ROL or the vehicle for 7 days by placing the rats on the rotating drums and measuring the time from the start of the acceleration period until the rat fell off the drum. A cut-off latency of $30 \mathrm{sec}$ was used for all rotarod assessments. The time that the animal remained on the rotarod was recorded and expressed as a percentage of its own baseline value.

Immunohistochemical staining. The rats were deeply anesthetised with an overdose of chloral hydrate $(10 \%, 0.3 \mathrm{ml} / 100 \mathrm{~g})$ and transcardially perfused with $200 \mathrm{ml}$ of $0.01 \mathrm{M} \mathrm{PBS}$ (pH 7.4), followed by $500 \mathrm{ml}$ of $4 \%$ paraformaldehyde in $0.1 \mathrm{M}$ phosphate buffer (PB, pH 7.4). The L4-L6 spinal cord segments with the spinal dorsal horn (SDH) were harvested and dehydrated in $30 \%$ sucrose at $4^{\circ} \mathrm{C}$. Transverse spinal sections (30- $\mu$ m-thick) were then cut using a cryostat (CM3050S; Leica, Wetzlar, Germany). For double immunofluorescence, the sections were incubated with rabbit anti-c-Fos (1:500; ab209794; Abcam, Cambridge, MA, USA), mouse anti-glial fibrillary acidic protein (GFAP) (1:400; \#3670; Cell Signaling Technology, Danvers, MA, USA) and goat anti-IBA-1 (1:500; MABN92; Millipore, Billerica, MA, USA) antibodies, followed by FITC-conjugated secondary antibodies [Alexa Fluor 594 donkey anti-rabbit IgG (A21207), Alexa Fluor 488 donkey anti-mouse IgG (A21202) and Alexa Fluor 488 donkey anti-goat IgG (A11055); 1:500; all from Invitrogen, Carlsbad, CA, USA] for $2 \mathrm{~h}$ at room temperature. The stained sections were observed and captured under a confocal laserscanning microscope (FV1000; Olympus, Tokyo, Japan).

Western blot analysis. The L4-L6 spinal cord segments were rapidly extracted from the anesthetized rats. The tissues were homogenized in lysis buffer (Bio-Rad Laboratories, Hercules, CA, USA) which contains a mixture of protease inhibitors and phenylmethylsulfonyl fluoride (Roche Diagnostics, Basel, Switzerland). Equivalent amounts of protein $(10 \mu \mathrm{l})$ were loaded and separated by $10 \%$ sodium dodecyl sulfate-polyacrylamide gel electrophoresis (SDS-PAGE; Bio-Rad Laboratories), and transferred onto PVDF membranes (Millipore). The membranes were incubated with the primary antibodies overnight at $4{ }^{\circ} \mathrm{C}$, followed by HRP-conjugated secondary antibodies for $2 \mathrm{~h}(1: 1,000$; \#7074 and \#7076; Cell Signaling Technology). The secondary antibody signal was detected by enhanced chemiluminescence (Millipore), and captured using the ChemiDoc XRS system (Bio-Rad Laboratories). The following primary antibodies were used: rabbit anti-pJNK (1:1,000; ab4821), rabbit anti-JNK (1:1,000; ab85139), rabbit anti-PDE4B (1:1,500; ab14611) (all from Abcam), mouse anti-GFAP (1:2,000; \#3670; Cell Signaling Technology), goat anti-IBA1 (1:1,000; MABN92; Millipore) and mouse anti- $\beta$-actin (1:10,000; A1978; Sigma-Aldrich).

Enzyme-linked immunosorbent assay (ELISA). The spinal cord tissues were collected according to the same method used for western blot analysis. The spinal cord tissues were homogenized in a pre-made lysis buffer which contained protease and phosphatase inhibitors. The protein samples of L4-5 spinal tissues were collected according to the same method used for western blot analysis. The spinal cord tissues were homogenized in a pre-made lysis buffer which contained protease and phosphatase inhibitors. Rat IL-1 $\beta$ (RLB00), IL-6 (R6000B), TNF- $\alpha$ 

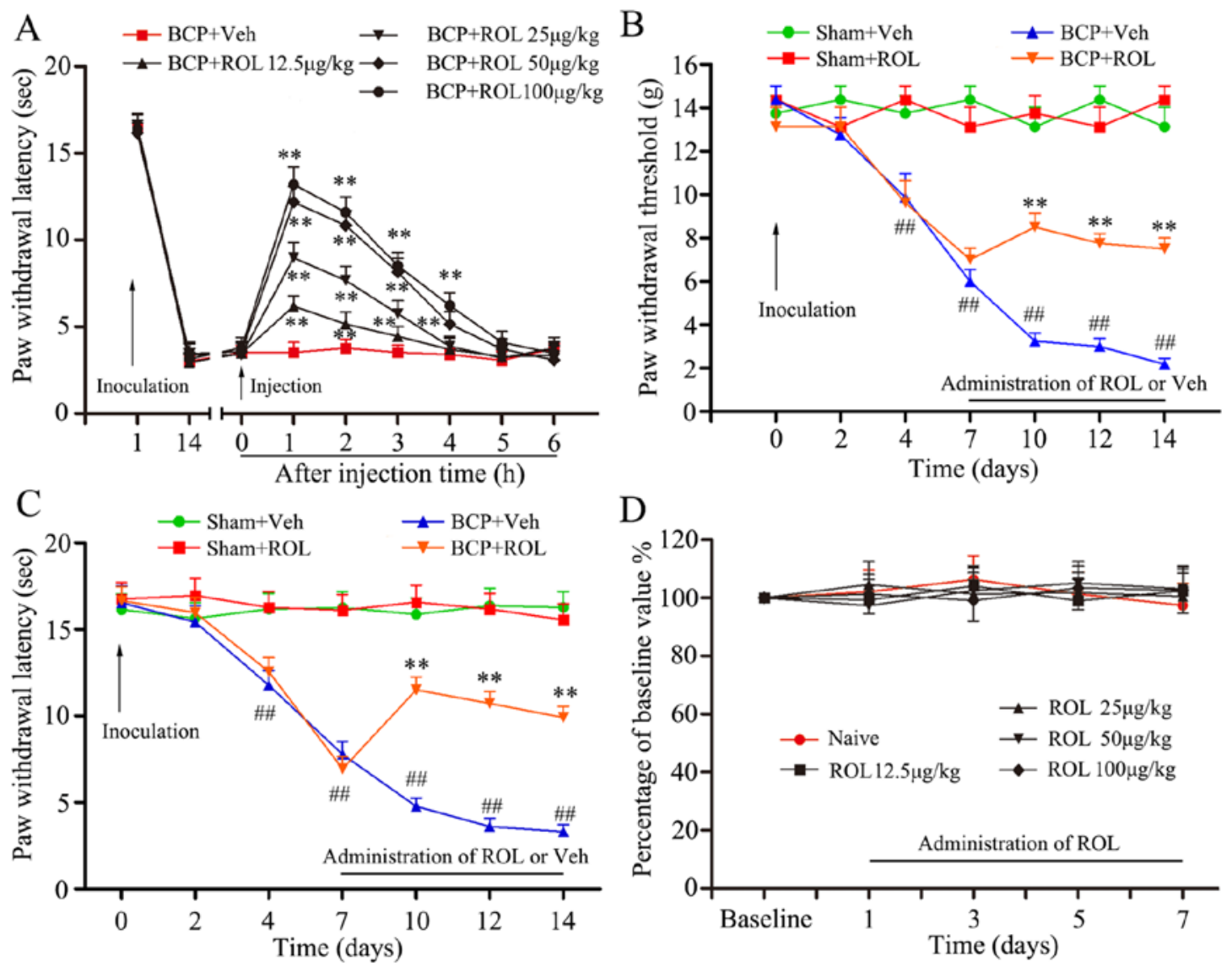

Figure 1. Rolipram (ROL) attenuates mechanical allodynia and thermal hyperalgesia in rats with bone cancer pain (BCP). (A) The intrathecal administration of ROL at doses of 12.5, 25, 50 and $100 \mu \mathrm{g} / \mathrm{kg}$ significantly attenuated BCP. (B) The paw mechanical withdrawal threshold and (C) the paw withdrawal latency were assessed in different groups of rats at the dose of $50 \mu \mathrm{g} / \mathrm{kg}$. (D) The effects of ROL at various doses on motor performance were examined by the rotarod test. ${ }^{* *}$ p $<0.01$ vs. $B C P+$ vehicle group; ${ }^{\# \#}$ p $<0.01$ vs. sham-operated group (sham). Eight rats were included in each group.

(RTA00) and CCL2 (MJE00) ELISA kits were purchased from R\&D Systems (Minneapolis, MN, USA). For each reaction in a 96-well plate, $100 \mu \mathrm{g}$ proteins from spinal tissue and standard were used. The expression levels of IL-1 $\beta$, IL-6, TNF- $\alpha$ and CCL 2 were determined by ELISA according to the manufacturer's instructions. According to the standard curve from each set of standard samples assayed, the concentration of all spinal samples was calculated.

Statistical analysis. All data were collected and analyzed by experienced researchers using blinded methods. Two-way ANOVA with Bonferroni post hoc tests were used for the nociceptive behavioral and rotarod data. One-way ANOVA with least significant difference (LSD) for post hoc analysis were used for immunohistochemistry ELISA and western blot analysis. All data are presented as the means \pm standard error of the mean (SEM). All statistical analyses were performed using GraphPad Prism version 5.01 for Windows (GraphPad Software, San Diego, CA, USA; www.graphpad.com). A value of $p<0.05$ was considered to indicate a statistically significant difference in all statistical analyses.

\section{Results}

Intrathecal administration of ROL attenuates mechanical allodynia and thermal hyperalgesia. The inoculation of tumor cells resulted in prominent mechanical allodynia and thermal hyperalgesia, as demonstrated in the $\mathrm{BCP}+$ vehicle group. In order to identify the potential analgesic effects of ROL in BCP, different doses of ROL, were i.t. injected on day 14 following tumor cell inoculation. Compared with $\mathrm{BCP}+$ vehicle group, the i.t. administration of ROL significantly elevated the PWL of the rats in a dose dependent manner $(\mathrm{p}<0.01)$, and the dose of $100 \mu \mathrm{g} / \mathrm{kg}$ did not exert a more prominent analgesic effect than the dose of $50 \mu \mathrm{g} / \mathrm{kg}$ (Fig. 1A). Thus, we selected $50 \mu \mathrm{g} /$ $\mathrm{kg}$ as the maximal dose for further experiments to determine the continuous analgesic effects of ROL. Subsequently, at dose of $50 \mu \mathrm{g} / \mathrm{kg}$, ROL was administered daily by i.t. injection from days 7 to 14 following tumor cell inoculation. The results from the behavioral test revealed that there was no apparent influence of the daily i.t. administration of ROL on paw withdrawal thresholds (PWTs) and PWLs in the sham + ROL group, indicating that the i.t. administration of ROL did not affect the basal pain threshold. However, compared with the $\mathrm{BCP}+$ vehicle group, the i.t. administration of ROL significantly elevated both the PWTs and PWLs, as shown by the Von Frey test $(\mathrm{p}<0.01$; Fig. 1B) and Hargreaves test $(\mathrm{p}<0.01$; Fig. 1C), respectively. From days 7 to 14 in the rats in the $\mathrm{BCP}+\mathrm{ROL}$ group, the i.t. administration of ROL attenuated mechanical allodynia and thermal hyperalgesia. For an ideal analgesic agent, fewer side-effects are always expected. Our results revealed that the daily i.t. administration of ROL did not affect 

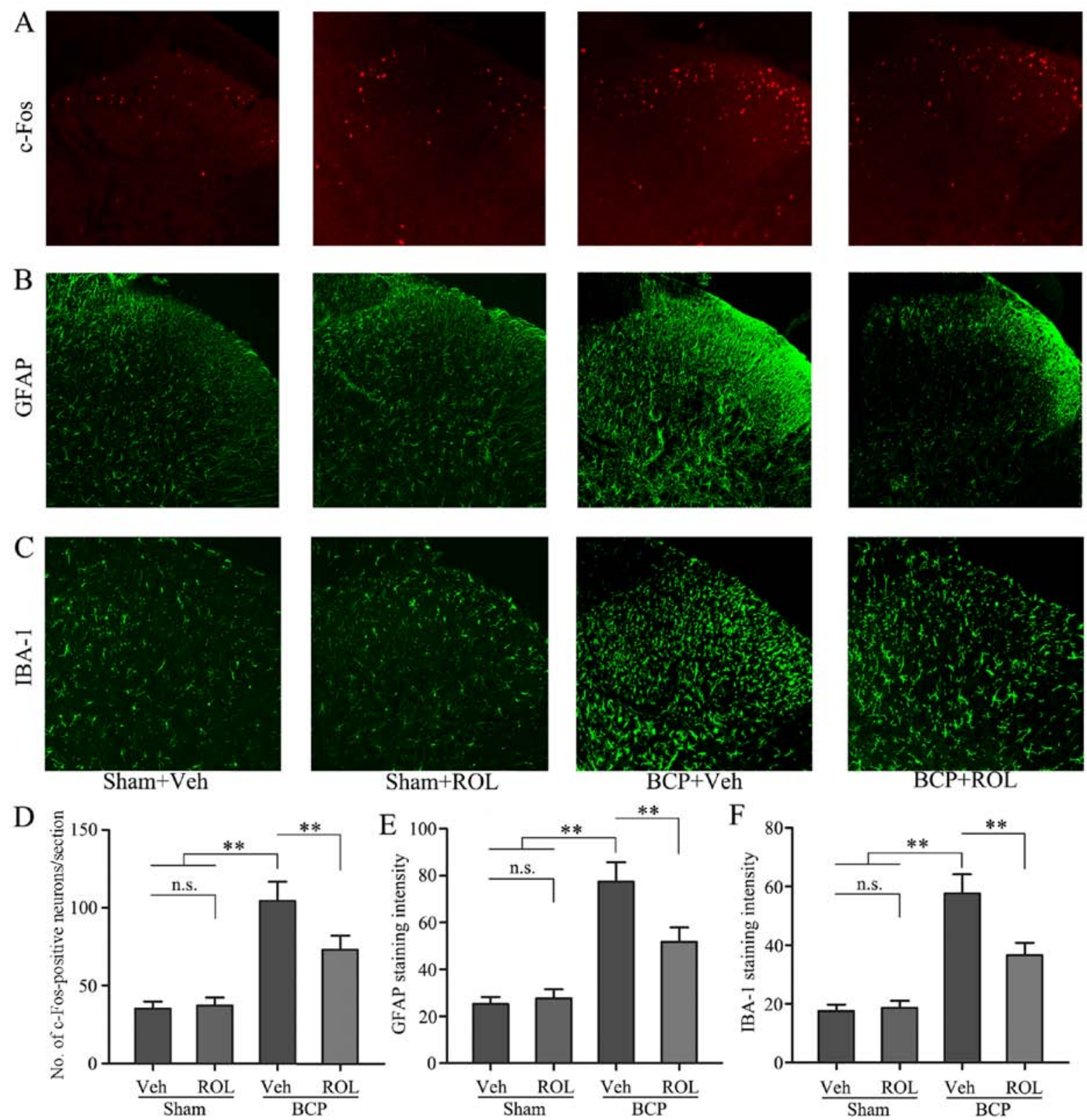

Figure 2. Rolipram (ROL) decreases neuron-astrocytic activation in the spinal dorsal horn (SDH). Immunohistochemical analysis of the expression of (A) c-Fos, (B) glial fibrillary acidic protein (GFAP) and (C) ionized calcium binding adapter molecule-1 (IBA-1). Quantification of the relative expression of (D) c-Fos, (E) GFAP and (F) IBA-1 in the SDH. Tissues were collected 14 days following inoculation. Original magnification, x200. " ${ }^{*}<0.01$; n.s., no significance; BCP, bone cancer pain; Veh, vehicle; Sham, sham-operated.. Four rats were included in each group.

the motor performance of the rats (ROL at the doses of $12.5,25$, 50 and $100 \mu \mathrm{g} / \mathrm{kg})(\mathrm{p}>0.05$; Fig. 1D), thus confirming that ROL exerts anti-allodynic and anti-hyperalgesic effects without any obvious measurable impairment on motor functions.

ROL inhibits PDE $4 B$ and neuron-astrocytic activation. Immunohistochemical staining was used to illustrate whether the analgesic effects of ROL at a dose of $50 \mu \mathrm{g} / \mathrm{kg}$ are accompanied by spinal neuron-astrocytic activation in BCP. Thus, we examined the expressoin of PDE4B, c-Fos, GFAP and IBA-1 on day 14 in the different groups. The upregulation of PDE4B was observed in the rats with BCP, and the i.t. administration of ROL significantly decreased PDE4B expression in the $\mathrm{BCP}+$ ROL group ( $<<0.01$; Fig. $3 \mathrm{~A}$ and $\mathrm{C})$. Compared with the sham-operated group, a significant increase in neuron- astrocytic activation, as indicated by the upregulation of c-Fos, GFAP and IBA-1 expression, predominantly in the superficial layers of the SDH, was observed in the rats with BCP following tumor cell inoculation. The consecutive daily i.t. administration of ROL significantly decreased the levels of c-Fos, GFAP and IBA-1, as indicated by immunohistochemistry (Fig. 2) and western blot analysis (Fig. 3) in the BCP + ROL group on day 14 , compared with the BCP + vehicle group. There was no apparent influence of the daily i.t. administration of ROL on c-Fos, GFAP and IBA-1 expression in the sham + ROL group [all p <0.01; c-Fos (Fig. 2A and D); GFAP (Figs. 2B and $\mathrm{E}$, and $3 \mathrm{~A}$ and $\mathrm{D}$ ); IBA-1 (Figs. 2C and F, and 3A and $\mathrm{E}$ )]. As expected, compared with the $\mathrm{BCP}+$ vehicle group, $\mathrm{ROL}$ significantly inhibited neuron-astrocytic activation on day 14 following tumor cell implantation. 

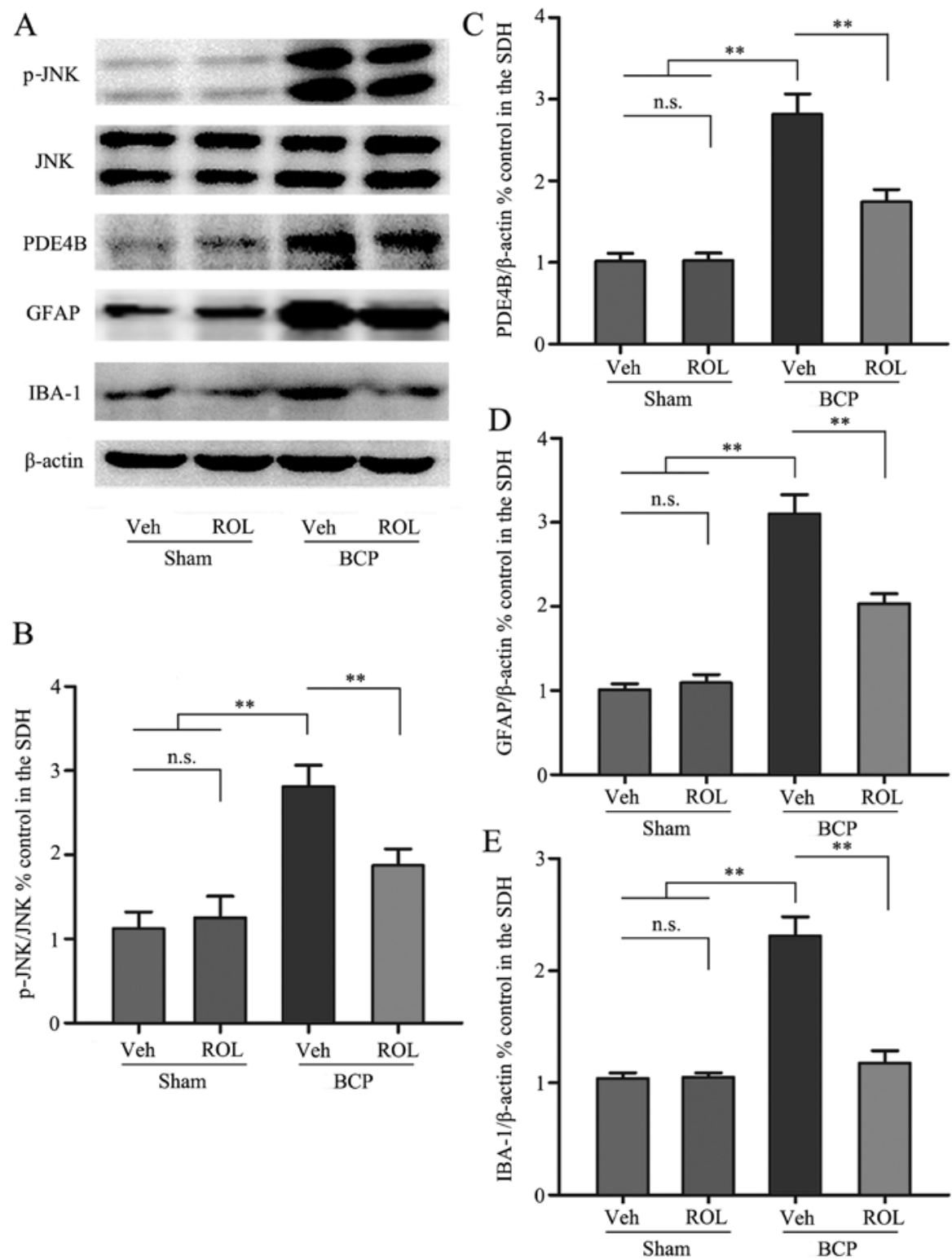

Figure 3. The spinal expression of p-c-Jun N-terminal kinase (JNK), phosphodiesterase 4B (PDE4B), glial fibrillary acidic protein (GFAP) and ionized calcium binding adapter molecule-1 (IBA-1) was inhibited by the intrathecal administration of rolipram (ROL). (A) Western blots showing the protein levels of p-JNK, PDE4B, GFAP and IBA-1. (B-E) Semi-quantitative assessment of the protein expression of p-JNK, PDE4B, GFAP and IBA-1 follwing ROL administration. Tissues were collected 14 days followig inoculation. ${ }^{* *}$ p $<0.01$; n.s., no significance; BCP, bone cancer pain; Veh, vehicle; Sham, sham-operated. Four rats were included in each group.

p-JNK activation is decreased by the administration of ROL. We further investigated whether the activation of p-JNK could be detected following the administration of ROL at a dose of $50 \mu \mathrm{g}$ / $\mathrm{kg}$. As shown by previous studies (12,27-29), almost all p-JNKpositive cells are located on GFAP-positive astrocytes in many types of pain models. In this study, western blot analysis was used to examine the effect of the i.t. administration of ROL on p-JNK expression, and the results revealed that the spinal expression of p-JNK was significantly enhanced in the BCP + vehicle group compared with the sham + vehicle group $(\mathrm{p}<0.01)$. However, ROL exerted a prominent inhibitory effect on $\mathrm{p}-\mathrm{JNK}$ expression as compared with the $\mathrm{BCP}+$ vehicle group $(\mathrm{p}<0.01)$. However, ROL did not affect $\mathrm{p}-\mathrm{JNK}$ expression in the sham + ROL group compared with the sham + vehicle group (Fig. 3A and B). These results suggest that the i.t. administration of ROL effectively inhibits the upregulation of $\mathrm{p}-\mathrm{JNK}$ in rats with $\mathrm{BCP}$.
ROL attenuates the upregulation of pro-inflammatory cytokines and CCL2 in rats with BCP. In order to investigate which possible intracellular signal transduction pathway plays a role in the analgesic effects of ROL at a dose of $50 \mu \mathrm{g} / \mathrm{kg}$ in rats with BCP, we detected the levels of the pro-inflammatory cytokines, IL-1 $\beta$, IL- 6 and TNF- $\alpha$, as well as those of CCL2 in the rats in each group. Compared with sham + vehicle group, a significant increase in the levels of IL- $1 \beta$, IL- 6, TNF- $\alpha$ and CCL2 was observed in the BCP + vehicle group. However, following the i.t. administration of ROL for 7 consecutive days, the expression levels of these above-mentioned factors were significantly inhibited in the $\mathrm{BCP}+\mathrm{ROL}$ group, compared with the $\mathrm{BCP}+$ vehicle group $(\mathrm{p}<0.01$; Fig. 4). Furthermore, the i.t. administration of ROL did not alter the expression levels of spinal pro-inflammatory cytokines and CCL2 in the sham + ROL group (Fig. 4). These results suggest that ROL 

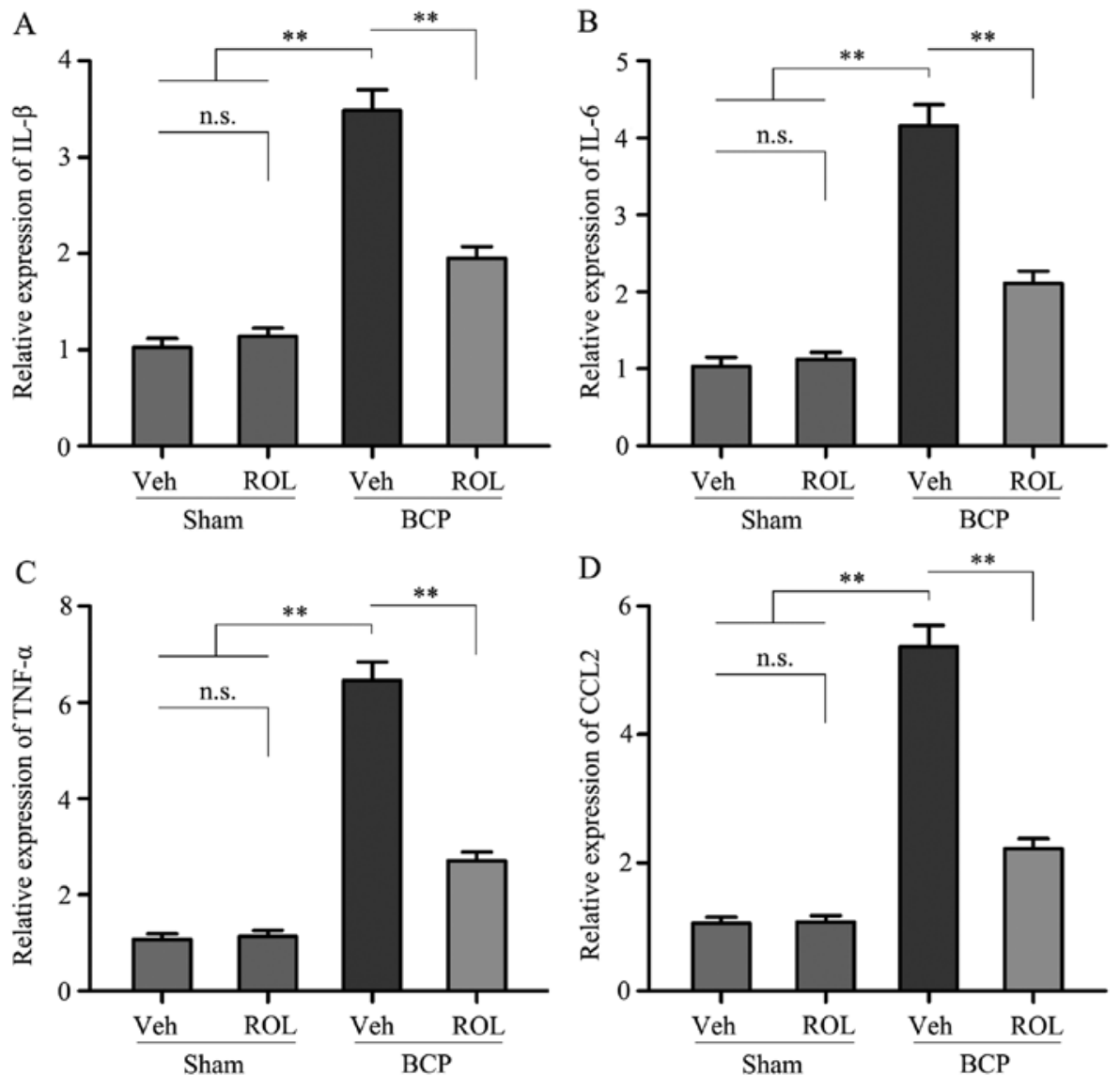

Figure 4. Rolipram (ROL) decreases spinal pro-inflammatory cytokine levels in rats with bone cancer pain (BCP). The levels of (A) interleukin (IL)-1 $\beta$, (B) IL-6, (C) tumor necrosis factor (TNF)- $\alpha$ and (D) chemokine (C-C motif) ligand 2 (CCL2) were examined by ELISA. Tissues were collected 14 days following inoculation. ${ }^{* *}$ p $<0.01$; n.s., no significance; Veh, vehicle; Sham, sham-operated. Four rats were included in each group.
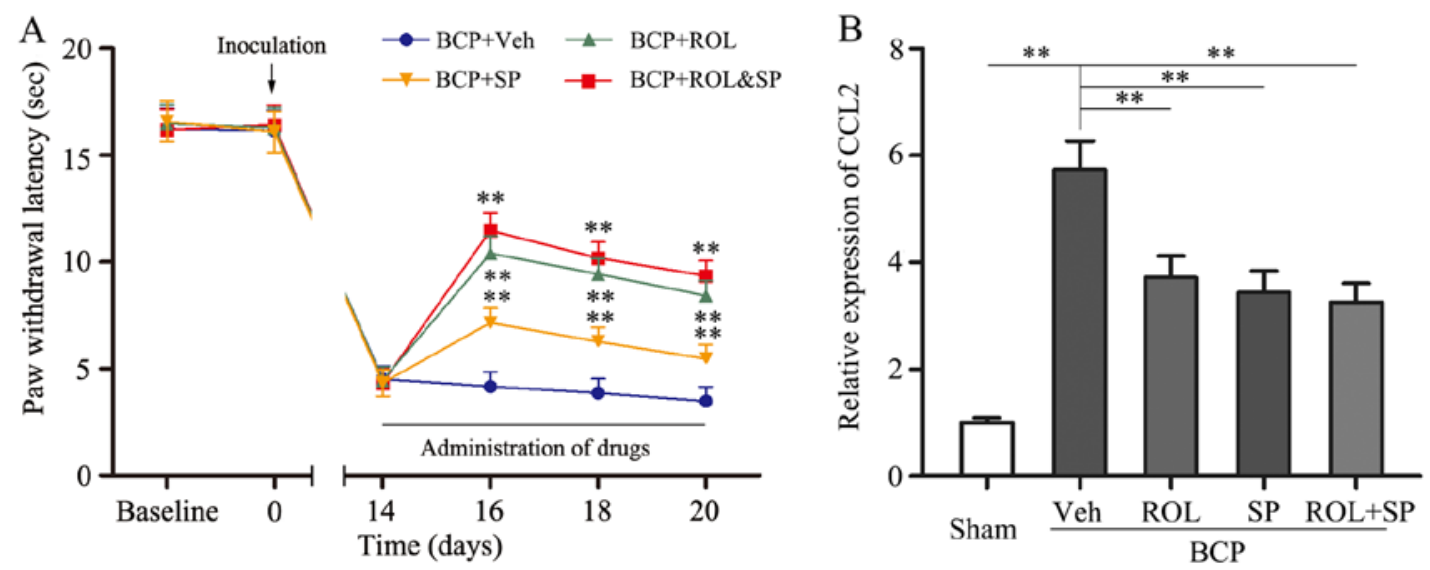

Figure 5. Co-administration of rolipram (ROL) and SP600125 attenuates thermal hyperalgesia and chemokine (C-C motif) ligand 2 (CCL2) expression in rats with bone cancer pain (BCP). The intrathecal administration of SP600125 significantly attenuated thermal hyperalgesia compared with the vehicle control. The co-administration of ROL and SP600125 did not exert more potent effects on (A) paw withdrawal latency and (B) CCL2 expression compared to the administration of ROL alone. Tissues were collected 20 days following inoculation. ${ }^{* *}$ p $<0.01 ;$ n.s., no significance; Veh, vehicle; Sham, sham-operated; SP, SP600125. Four rats were included in each group.

effectively inhibits the upregulation of pro-inflammatory cytokines and CCL2 in rats with BCP.

The analgesic effects of ROL are mediated via the inhibition of the CCL2/JNK pathway in astrocytes in rats with $B C P$. It was found that the inhibition of neuron-astrocytic activation by ROL contributes to BCP; however, we wished to determine which intracellular signal transduction pathway contributes to these effects. SP600125, a specific JNK inhibitor, was i.t. injected into the spinal cord of the rats. The consecutive i.t. administration of SP600125 (5 $\mu \mathrm{g})$ for 7 days significantly attenuated thermal hyperalgesia compared with the BCP + vehicle group ( $<<0.01$; Fig. 5). Following the co-administration of ROL $(50 \mu \mathrm{g} / \mathrm{kg})$ and SP600125 $(5 \mu \mathrm{g})$ 


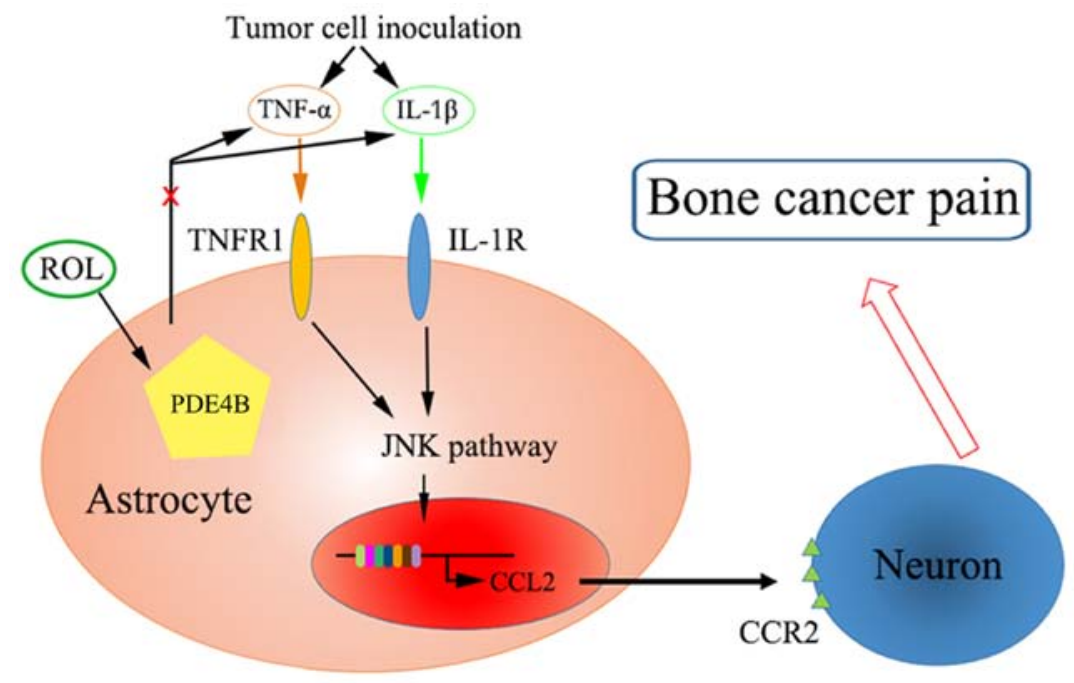

Figure 6. Schematic diagram of the mechanisms through which rolipram (ROL) attenuates pain behavior in bone cancer pain. TNFR1, tumor necrosis factor receptor 1.

to the rats with $\mathrm{BCP}$, no significant difference was observed in the analgesic effects compared to the BCP + ROL group. Furthermore, similar results were observed with CCL2 expression; the co-administration of ROL and SP600125 did not exert any further inhibitory effects on CCL2 expression in the rats with BCP than the administration of ROL $(50 \mu \mathrm{g} / \mathrm{kg})$ alone (Fig. 5). Thus, the analgesic effects of ROL appear to be dependent mainly on the inhibition of the activation of the CCL2/JNK pathway.

\section{Discussion}

ROL, a well-characterized PDE4 inhibitor, is an effective choice in the treatment of respiratory diseases, such as chronic obstructive pulmonary disease (COPD) and asthma, and has also been widely investigated in research field of spinal cord injury, Alzheimer's disease, memory deficits and analgesia (30-33). To the best of our knowledge, this is the first study to demonstrate the beneficial analgesic effects of ROL in a model of BCP. In this study, we demonstrated that progressive nociceptive behavior could be induced by tumor cell inoculation with significant neuron-astrocytic activation in the SDH. ROL exerted a potent analgesic effect as a single injection or as a continuous injection, and significantly attenuated nociceptive behaviors. Additionally, the neuron-astrocytic activation and the JNK pathway were markedly inhibited, and the release of pro-inflammatory cytokines (IL-1 $\beta$, IL-6 and TNF- $\alpha$ ) was also suppressed. We also found that the combined analgesic effects of ROL and the JNK inhibitor, SP600125, on nociceptive behaviors did not differ from those observed with ROL administration alone. Finally, the expression of CCL2, which is known to be the downstream chemokine of the JNK pathway, was significantly decreased by treatment with ROL. Based on the above-mentioned evidence, our results indicate that ROL, a potential specific candidate for the management of pro-inflammatory factor production and the development of BCP, can attenuate nociceptive behavior wholly or mainly by inhibiting the activation of the JNK/CCL2 signaling pathway in rats with BCP.
The main function of PDE4 is degrading the cAMP phosphodiester bond and terminating its functions $(13,34)$. It has been demonstrated that PDE4 is most predominantly expressed in glial cells and immune cells in the CNS, and inhibitors of PDE4 exhibit anti-depressant, anti-asthmatic, anti-inflammatory properties (35). In particular, there is evidence to indicate that PDE4B plays a crucial role in neuro-inflammation. PDE4B has been shown to be upregulated with pain behavior following L5 nerve ligation in a model of neuropathic pain, and the i.t. administration of PDE4B siRNA was shown to attenuate pain behavior with the inhibition of PDE4B (36). Furthermore, ROL has ability to act upon the CNS and increase the level of the cAMP predominantly in both nerve and immune tissues, and then activate protein kinase A (PKA) (37). Activated PKA can suppress the activation of nuclear factor- $\kappa \mathrm{B}$ and the increased activity of N-methyl-D-aspartate receptor, and can then decrease the production of many pro-inflammatory cytokines, such as inflammatory cytokines (TNF- $\alpha$, IL-1 and IL-6), chemotaxis and cytotoxicity. However, the increased expression of pro-inflammatory cytokines (IL-1 $\beta$, IL-6 and $\mathrm{TNF}-\alpha$ ) is closely associated with many types of pain models (38), and plays an important role in the progression and maintenance of pain. In this study, an increase in the levels of IL-1 $\beta$, IL- 6 and TNF- $\alpha$ was detected in rats with BCP with the activation of astrocytes and microglia, and ROL attenuated hyperalgesia by inhibiting the levels of IL-1 $\beta$, IL-6 and $\mathrm{TNF}-\alpha$, and subsequently, astrocyte and microglial activation was also suppressed. In addition, ROL had been reported to exert analgesic effects in chemotherapy-induced neuropathic pain in rats (17). Based on the above-mentioned evidence, we proved that ROL inhibited the increase in the levels of IL-1 $\beta$, IL-6, TNF- $\alpha$ in rats with BCP, and then decreased neuroglial activation, exerting an analgesic effect. Therefore, ROL may be a potent candidate analgesic agent for $\mathrm{BCP}$.

It is known to all that spinal neuronal sensitization may be dominant in the pain 'initiation phase', while neuroglial cells, particularly astrocytes play a pivotal role in the maintenance of BCP, acting as the main immune cells in the CNS $(23,39)$. 
Activated neuroglia produce numerous mediators, such as pro-inflammatory cytokines and chemokines which results in the cascading activation of surrounding neuroglia that enhance neuronal activity; furthermore, these activated pro-inflammatory cytokines and chemokines can maintain the sustained activation of astrocytes (40). It has recently been found that blocking the activation of spinal astrocytes with a glial metabolic inhibitor impedes the exaggerated pain induced by peripheral tissue inflammation, nerve injury and spinal cord impairment (41). In the present study, we observed the alleviation of nociceptive behaviors following the administration of ROL. In addition, we found that the expression of GFAP and IBA-1 in the SDH was significantly reduced, and this result indicated that astrocytic and microglial activation was suppressed. According to these results, we suggest that the analgesic effects of ROL may be possibly associated with the suppression of neuroglial cell activation.

JNK, a key intracellular kinase of the MAPK pathway, plays an important role in chronic pain and astrocytic activation $(42,43)$. The overexpression of $\mathrm{p}-\mathrm{JNK}$ can be detected in many types of pain models. In this study, following the i.t. injection of SP600125, a JNK selective inhibitor, thermal hyperalgesia was significantly attenuated. Additionally, another JNK inhibitor, D-JNKI-1, has also shown potential analgesic effects in spinal nerve ligation (SNL)-induced neuropathic pain (44). In this study, tumor cell inoculation promoted the increase in $\mathrm{p}-\mathrm{JNK}$ expression in the SDH, and the increase in p-JNK expression was inhibited by the i.t. injection of ROL with the alleviation of nociceptive behaviors. Subsequently, the co-administration of ROL and SP600125 did not exert more potent analgesic effects than the administration of ROL alone. These results suggest that the analgesic effects of ROL may be associated with the inhibition of spinal p-JNK expression. A number of studies have confirmed that pro-inflammatory cytokines, particularly IL-1 $\beta$, IL- 6 and TNF- $\alpha$, induced by nerve injury and inflammation, are essential initiators for the spinal activation of p-JNK in astrocytes underlying the maintenance of many types of chronic pain (45-47). Similarly, in our study, the levels of pro-inflammatory cytokines (IL-1 $\beta$, IL- 6 and $\mathrm{TNF}-\alpha$ ) in the SDH were decreased by the i.t. injection of ROL, indicating that the analgesic effects of ROL are associated with the inhibition of spinal p-JNK activation via the suppression of the inflammatory cascade reaction.

It has been proven that CCL2 is produced by astrocytes via the JNK-mediated signaling pathway in neuropathic pain and subsequently mediates this pain via CCR 2 receptors in neurons (12). The administration of a JNK inhibitor (D-JNKI-1) has been shown to inhibit the production of CCL 2 in TNF- $\alpha$ or IL-1 $\beta$-induced inflammatory conditions in neuropathic pain (44). Additionally, in mice, the overexpression of CCL2 is mainly found in astrocytes and enhances nociceptive responses (48). In this study, the analgesic effects of ROL were mediated partly by the downregulation of CCL2. Tumor cell inoculation increased the expression of pro-inflammatory cytokines (IL-1 $\beta$, IL-6 and TNF- $\alpha$ ) and PDE4B expression, and was associated with the activation of the JNK/CCL2 pathway. We hypothesize that ROL attenuated BCP through the following mechanisms: the administration of ROL antagonized the changes in astrocyte and microglial cell morphology and reduced pro-inflammatory cytokine production. Subsequently,
JNK activation was inhibited accompanied by the decrease of astrocyte-secreted CCL2, and finally, nociceptive responses were attenuated by the reduction of the sensitivity of neurons (Fig. 6).

The i.t. administration if ROL exerted potent anti-inflammatory and anti-nociceptive effects in rats with BCP. However, further investigations and clinical testing are required to confirm our findings. ROL exerted long-acting analgesic effects. Thus, this notable characteristic makes ROL good candidate for use in the long-term treatment of chronic pain, particularly BCP. Possibly, in the beginning and/or advanced stage of chronic pain treatment, other short-onset analgesics, such as morphine, can be used simultaneously with ROL. After a few days, ROL or PDE4 inhibitor could be used as the only treatment, thereby avoiding the side-effects of long-term morphine treatment in clinical practice. This may be a novel strategy for the treatmetn of chronic pain.

In conclusion, the present study suggested that the i.t. administration of ROL attenuated nociceptive behaviors in BCP rats through the inhibition of pro-inflammatory cytokines (IL-1 $\beta$, IL-6 and TNF- $\alpha$ ). Furthermore, the suppression of the astrocyte-related JNK/CCL2 pathway was involved in the analgesic effects of ROL. Thus, we suggest that ROL may be a potential specific candidate with which to regulate and attenuate the development of BCP. However, there is still a great barrier between animal reasearch and human application. Further experimental studies on the effective safe dosage for human, pharmacokinetics and specific activation pathways are warranted in order to determine the effective use of ROL in the treatment and management of chronic pain.

\section{Acknowledgements}

This study was supported by grants from the National Natural Science Foundation of China (nos. 81371112 and 81171050) and the Program for Shaanxi Province Key Research Team of Science and Technology Innovation (no. 2012KCT-14).

\section{References}

1. Lozano-Ondoua AN, Symons-Liguori AM and Vanderah TW: Cancer-induced bone pain: Mechanisms and models. Neurosci Lett 557: 52-59, 2013.

2. Bennett MI, Rayment C, Hjermstad M, Aass N, Caraceni A and Kaasa S: Prevalence and aetiology of neuropathic pain in cancer patients: A systematic review. Pain 153: 359-365, 2012.

3. Mantyh PW: Cancer pain and its impact on diagnosis, survival and quality of life. Nat Rev Neurosci 7: 797-809, 2006.

4. Ji RR, Berta T and Nedergaard M: Glia and pain: Is chronic pain a gliopathy? Pain 154 (Suppl 1): S10-S28, 2013.

5. Yang Y, Li H, Li TT, Luo H, Gu XY, Lü N, Ji RR and Zhang YQ: Delayed activation of spinal microglia contributes to the maintenance of bone cancer pain in female Wistar rats via P2X7 receptor and IL-18. J Neurosci 35: 7950-7963, 2015.

6. Remeniuk B, Sukhtankar D, Okun A, Navratilova E, Xie JY, King T and Porreca F: Behavioral and neurochemical analysis of ongoing bone cancer pain in rats. Pain 156: 1864-1873, 2015.

7. Oprée A and Kress M: Involvement of the proinflammatory cytokines tumor necrosis factor-alpha, IL-1 beta, and IL-6 but not IL-8 in the development of heat hyperalgesia: Effects on heat-evoked calcitonin gene-related peptide release from rat skin. J Neurosci 20: 6289-6293, 2000.

8. Chichorro JG, Lorenzetti BB and Zampronio AR: Involvement of bradykinin, cytokines, sympathetic amines and prostaglandins in formalin-induced orofacial nociception in rats. $\mathrm{Br} \mathrm{J}$ Pharmacol 141: 1175-1184, 2004. 
9. Zhou L, Huang J, Gao J, Zhang G and Jiang J: NMDA and AMPA receptors in the anterior cingulate cortex mediates visceral pain in visceral hypersensitivity rats. Cell Immunol 287: 86-90, 2014.

10. Guo W, Miyoshi K, Dubner R, Gu M, Li M, Liu J, Yang J, Zou S, Ren K, Noguchi K and Wei F: Spinal 5-HT3 receptors mediate descending facilitation and contribute to behavioral hypersensitivity via a reciprocal neuron-glial signaling cascade. Mol Pain 10: 35, 2014.

11. Wang XW, Hu S, Mao-Ying QL, Li Q, Yang CJ, Zhang H, Mi WL, Wu GC and Wang YQ: Activation of c-jun N-terminal kinase in spinal cord contributes to breast cancer induced bone pain in rats. Mol Brain 5: 21, 2012.

12. Tang J, Zhu C, Li ZH, Liu XY, Sun SK, Zhang T, Luo ZJ, Zhang H and LiWY: Inhibition of the spinal astrocytic JNK/MCP-1 pathway activation correlates with the analgesic effects of tanshinone IIA sulfonate in neuropathic pain. J Neuroinflammation 12: 57, 2015.

13. Houslay MD and Adams DR: PDE4 cAMP phosphodiesterases: Modular enzymes that orchestrate signalling cross-talk, desensitization and compartmentalization. Biochem J 370: 1-18, 2003.

14. Pearse DD and Hughes ZA: PDE4B as a microglia target to reduce neuroinflammation. Glia 64: 1698-1709, 2016.

15. Kumar A, Jain NK and Kulkarni SK: Analgesic and antiinflammatory effects of phosphodiesterase inhibitors. Indian $\mathbf{J}$ Exp Biol 38: 26-30, 2000

16. Francischi JN, Yokoro CM, Poole S, Tafuri WL, Cunha FQ and Teixeira MM: Anti-inflammatory and analgesic effects of the phosphodiesterase 4 inhibitor rolipram in a rat model of arthritis. Eur J Pharmacol 399: 243-249, 2000.

17. Kim HK, Kwon JY, Yoo C and Abdi S: The analgesic effect of rolipram, a phosphodiesterase 4 inhibitor, on chemotherapyinduced neuropathic pain in rats. Anesth Analg 121: 822-828, 2015.

18. Rock EM, Benzaquen J, Limebeer CL and Parker LA: Potential of the rat model of conditioned gaping to detect nausea produced by rolipram, a phosphodiesterase-4 (PDE4) inhibitor. Pharmacol Biochem Behav 91: 537-541, 2009.

19. Sanna MD, Ghelardini C and Galeotti N: Blockade of the spinal BDNF-activated JNK pathway prevents the development of antiretroviral-induced neuropathic pain. Neuropharmacology 105: 543-552, 2016.

20. Zhang Q, Wang J, Duan MT, Han SP, Zeng XY and Wang JY: $\mathrm{NF}-\kappa \mathrm{B}, \mathrm{ERK}, \mathrm{p} 38$ MAPK and JNK contribute to the initiation and/or maintenance of mechanical allodynia induced by tumor necrosis factor-alpha in the red nucleus. Brain Res Bull 99: 132-139, 2013.

21. Hu XM, Liu YN, Zhang HL, Cao SB, Zhang T, Chen LP and Shen W: CXCL12/CXCR4 chemokine signaling in spinal glia induces pain hypersensitivity through MAPKs-mediated neuroinflammation in bone cancer rats. J Neurochem 132: 452-463, 2015

22. Wu HH, Yin JB, Zhang T, Cui YY, Dong YL, Chen GZ and Wang W: Inhibiting spinal neuron-astrocytic activation correlates with synergistic analgesia of dexmedetomidine and ropivacaine. PLoS One 9: e92374, 2014.

23. Liu S, Liu YP, Song WB and Song XJ: EphrinB-EphB receptor signaling contributes to bone cancer pain via Toll-like receptor and proinflammatory cytokines in rat spinal cord. Pain 154: 2823-2835, 2013.

24. Dixon WJ: Efficient analysis of experimental observations. Annu Rev Pharmacol Toxicol 20: 441-462, 1980.

25. Hargreaves K, Dubner R, Brown F, Flores C and Joris J: A new and sensitive method for measuring thermal nociception in cutaneous hyperalgesia. Pain 32: 77-88, 1988.

26. Hamm RJ, Pike BR, O'Dell DM, Lyeth BG and Jenkins LW: The rotarod test: An evaluation of its effectiveness in assessing motor deficits following traumatic brain injury. J Neurotrauma 11: 187-196, 1994.

27. Mei XP, Zhang H, Wang W, Wei YY, Zhai MZ, Wang W, Xu LX and Li YQ: Inhibition of spinal astrocytic c-Jun N-terminal kinase (JNK) activation correlates with the analgesic effects of ketamine in neuropathic pain. J Neuroinflammation 8: 6, 2011.

28. Gao YJ and Ji RR: Activation of JNK pathway in persistent pain. Neurosci Lett 437: 180-183, 2008.

29. Li ZY, Zhang YP, Zhang J, Zhang SB, Li D, Huang ZZ and Xin WJ: The possible involvement of JNK activation in the spinal dorsal horn in bortezomib-induced allodynia: the role of TNF- $\alpha$ and IL-1 $\beta$. J Anesth 30: 55-63. 2016.
30. Wang ZZ, Zhang Y, Liu YQ, Zhao N, Zhang YZ, Yuan L, An L, Li J, Wang XY, Qin JJ, et al: RNA interference-mediated phosphodiesterase 4D splice variants knock-down in the prefrontal cortex produces antidepressant-like and cognition-enhancing effects. Br J Pharmacol 168: 1001-1014, 2013.

31. García-Osta A, Cuadrado-Tejedor M, García-Barroso C, Oyarzábal J and Franco R: Phosphodiesterases as therapeutic targets for Alzheimer's disease. ACS Chem Neurosci 3: 832-844, 2012.

32. Wang ZZ, Yang WX, Zhang Y, Zhao N, Zhang YZ, Liu YQ, Xu Y, Wilson SP, O'Donnell JM, Zhang HT and Li YF: Phosphodiesterase-4D knock-down in the prefrontal cortex alleviates chronic unpredictable stress-induced depressive-like behaviors and memory deficits in mice. Sci Rep 5: 11332, 2015.

33. Huang Z and Mancini JA: Phosphodiesterase 4 inhibitors for the treatment of asthma and COPD. Curr Med Chem 13: 3253-3262, 2006.

34. Bolger GB, Dunlop AJ, Meng D, Day JP, Klussmann E, Baillie GS, Adams DR and Houslay MD: Dimerization of cAMP phosphodiesterase-4 (PDE4) in living cells requires interfaces located in both the UCR1 and catalytic unit domains. Cell Signal 27: 756-769, 2015.

35. Christiansen SH, Selige J, Dunkern T, Rassov A and Leist M: Combined anti-inflammatory effects of $\beta 2$-adrenergic agonists and PDE4 inhibitors on astrocytes by upregulation of intracellular cAMP. Neurochem Int 59: 837-846, 2011.

36. Ji Q, Di Y, He X, Liu Q, Liu J, Li W and Zhang L: Intrathecal injection of phosphodiesterase 4B-specific siRNA attenuates neuropathic pain in rats with L5 spinal nerve ligation. Mol Med Rep 13: 1914-1922, 2016.

37. Nunes AR, Sample V, Xiang YK, Monteiro EC, Gauda E and Zhang J: Effect of oxygen on phosphodiesterases (PDE) 3 and 4 isoforms and PKA activity in the superior cervical ganglia. Adv Exp Med Biol 758: 287-294, 2012.

38. Perez-Aso M, Montesinos MC, Mediero A, Wilder T, Schafer PH and Cronstein B: Apremilast, a novel phosphodiesterase 4 (PDE4) inhibitor, regulates inflammation through multiple cAMP downstream effectors. Arthritis Res Ther 17: 249, 2015.

39. Old EA, Clark AK and Malcangio M: The role of glia in the spinal cord in neuropathic and inflammatory pain. Handb Exp Pharmacol 227: 145-170, 2015.

40. Alfonso Romero-Sandoval E and Sweitzer S: Nonneuronal central mechanisms of pain: Glia and immune response. Prog Mol Biol Transl Sci 131: 325-358, 2015.

41. Romero-Alejo E, Puig MM and Romero A: Inhibition of astrocyte activation is involved in the prevention of postoperative latent pain sensitization by ketamine and gabapentin in mice. J Pharmacol Pharmacother 7: 22-24, 2016.

42. Li W, Zhang Y, Xing $\mathrm{C}$ and Zhang M: Tanshinone IIA represses inflammatory response and reduces radiculopathic pain by inhibiting IR AK- 1 and NF- $\mathrm{NB} / \mathrm{p} 38 / \mathrm{JNK}$ signaling. Int Immunopharmacol 28: 382-389, 2015.

43. Gao YJ, Zhang L, Samad OA, Suter MR, Yasuhiko K, Xu ZZ, Park JY, Lind AL, Ma Q and Ji RR: JNK-induced MCP-1 production in spinal cord astrocytes contributes to central sensitization and neuropathic pain. J Neurosci 29: 4096-4108, 2009.

44. Lu Y, Jiang BC, Cao DL, Zhang ZJ, Zhang X, Ji RR and Gao YJ: TRAF6 upregulation in spinal astrocytes maintains neuropathic pain by integrating TNF- $\alpha$ and IL-1 $\beta$ signaling. Pain 155: 2618-2629, 2014.

45. Lu C, Liu Y, Sun B, Sun Y, Hou B, Zhang Y, Ma Z and Gu X: Intrathecal injection of JWH-015 attenuates bone cancer pain via time-dependent modification of pro-inflammatory cytokines expression and astrocytes activity in spinal cord. Inflammation 38: 1880-1890, 2015.

46. Pillarisetti S: Targeting interleukin-1 $\beta$ for pain. CNS Neurol Disord Drug Targets 10: 571-575, 2011.

47. Narita M, Shimamura M, Imai S, Kubota C, Yajima Y, Takagi T, Shiokawa M, Inoue T, Suzuki M and Suzuki T: Role of interleukin-1beta and tumor necrosis factor-alpha-dependent expression of cyclooxygenase-2 mRNA in thermal hyperalgesia induced by chronic inflammation in mice. Neuroscience 152: 477-486, 2008

48. Pevida M, González-Rodríguez S, Lastra A, García-Suárez O, Hidalgo A, Menéndez L and Baamonde A: Involvement of spinal chemokine CCL2 in the hyperalgesia evoked by bone cancer in mice: A role for astroglia and microglia. Cell Mol Neurobiol 34: 143-156, 2014. 